\title{
UTILIZAÇÃO DE PLANTAS MEDICINAIS EM UM MUNICÍPIO INSERIDO NO BIOMA PAMPA BRASILEIRO
}

\author{
Denise BRIÃO ${ }^{1}$ \\ Leonardo Luis ARTICO ${ }^{1,2}$ \\ Luís Fernando Paiva LÍMA ${ }^{1,3}$ \\ Ana Paula Simões MENEZES ${ }^{1,2}$
}

\begin{abstract}
${ }^{1}$ Centro de Ciências da Saúde da Universidade da Região da Campanha (URCAMP), Avenida Tupy Silveira, n. 2099, Bagé, RS, 96400100

${ }^{2}$ Instituto Biotecnológico de Reprodução Vegetal (INTEC)/ URCAMP, Rua. Gen. Flores da Cunha, n. 310, Bagé, RS, 96400-350. Autor para correspondência: anapaulasime@ gmail.com .

${ }^{3}$ Instituto Federal Farroupilha - Campus São Vicente do Sul, Rua 20 de Setembro, s/n,São Vicente do Sul, RS, 97420000 .
\end{abstract}

Recebido em: 08/12/2015 - Aprovado em: 16/07/2016 - Disponibilizado em: 18/12/2016

\section{RESUMO:}

Este estudo objetivou verificar o perfil de utilização de plantas medicinais por moradores do município de Hulha Negra, com população predominantemente de origem alemã e que está inserido no Bioma Pampa (RS), Brasil. O cálculo amostral compreendeu a população residente da zona urbana (2.909 mil habitantes). e resultou em valor de $\mathrm{N}=344$ indivíduos. Visitas domiciliares foram realizadas em dias úteis da semana entre agosto a novembro de 2011. Aplicou-se um questionário com abordagem sócio-demográfica e utilização de plantas medicinais, com base em condição de saúde. A categorização dos problemas de saúde à partir do motivo de uso das plantas foi realizada por sistemas orgânicos. As plantas foram classificads como nativas do Bioma Pampa e exóticas ao bioma. Foi possível acessar 137 moradores, sendo 128 usuários de plantas medicinais. Destes, $69,5 \%$ representados por mulheres, 39,8\% com idades superior a 50 anos e 46,8\% com nove anos de estudo ou mais. As famílias botânicas mais prevalentes foram Asteraceae (21\%) e Laminaceae (16\%), sendo a Macela a planta mais citada (55). Os principais motivos de uso de plantas medicinais foram os sintomas associados ao sistema digestivo (29\%), as condições de dor e inflamação (24\%) e ligadas ao sistema cardiovascular (14\%). Do total de espécies identificadas (67) $38,8 \%$ corresponderam a plantas nativas do Bioma Pampa.

Palavras-chave: Plantas medicinais; Etnobotânica; Bioma Pampa; Brasil.

\section{ABSTRACT:}

This study aimed to verify the profile of use of medicinal plants by residents of Hulha Negra city, with a population predominantly of German origin and is inserted into the Biome Pampa (RS), Brazil. The sample size calculation included the resident population of the urban area (2.909 million inhabitants) and resulted in value of $\mathrm{N}=344$ individuals. Home visits were carried out on weekdays between August and November, 2011. It was used a questionnaire with socio-demographic questions and on the use of medicinal plants, based on health condition. The categorization of health problems from the use of reason plants by respondents was performed by organ systems. The plants were classified as native's biome Pampa, and exotic to the biome.. It is accessed 137 residents at their homes, and 128 users of medicinal plants. Of these, $69.5 \%$ represented by women, $39.8 \%$ over the age of 50 and $46.8 \%$ with nine years of schooling or more. The most prevalent 
botanical families were Asteraceae (21\%) and Laminaceae (16\%), the Macela being the most mentioned plant (55). The main reasons for the use of medicinal plants were the symptoms associated with the digestive system (29\%), conditions of pain and inflammation (24\%) and connected to the cardiovascular system (14\%). The total of identified species (67) 38,8\% were native plants of the Pampa Biome.

Keywords: Medicinal plants; Ethnobotany; Biome Pampa; Brazil.

\section{Introdução}

O uso de plantas medicinais para fins de tratamento e prevenção de enfermidades, é considerado uma das mais antigas formas de prática medicinal na humanidade, tornando-se o primeiro recurso terapêutico utilizado por povos primitivos (Tomazzoni, Negrelle \& Centa, 2006; Oliveira \& Menini Neto, 2012; Alves \& Povh, 2013). O resgate do conhecimento tradicional aliado à ciência estimula a conservação dos recursos vegetais e o desenvolvimento sustentável de uma comunidade (Kruel, Silva \& Pinheiro, 2005). Entretanto, apesar da grande evolução da medicina alopática, vários registros de procedimentos clínicos utilizam plantas com potencial farmacológico como forma alternativa ou adicional no tratamento de doenças (Veiga Jr. et al, 2005). Nesse sentido, o emprego destas para a promoção e recuperação da saúde tem evoluído ao longo dos tempos, não estando esta prática restritas ao meio rural, mas inserido no contexto urbano como fonte alternativa ou complementar da medicina convencional (Simões et al. 1998; Lorenzi \& Matos, 2008).

O Rio Grande do Sul (RS) é um estado com grande diversidade cultural, resultado da imigração de comunidades portuguesas, espanholas, africanas, italianas, alemãs, dentre outras, responsáveis pela implementação e adaptação de espécies medicinais na região (Koch, 2000). Dessa forma, a diversidade de grupos étnicos oportunizaram o uso de plantas medicinais com base no saber popular, proporcionando subsídios para um maior conhecimento da flora medicinal regional. Segundo Giulietti et al. (2005) apesar dos avanços tecnológicos e do incentivo ao estudos da região dos Campos Sulinos (situados no sul do Brasil), o conhecimento minucioso dos recursos naturais e sobre a etnobotânica na região ainda são escassos, visto que o Estado do Rio Grande do Sul (RS) contempla dois biomas, denominados: Bioma Pampa, correspondente à metade sul do RS e a Mata Atlântica, que inclui as áreas de campos no Planalto SulBrasileiro, representadas pela região norte do RS. O Bioma Pampa apresenta extensão territorial de cerca de 17,64 milhões de hectares, representando cerca de $63 \%$ de toda a área do estado do RS (Overbeck et al., 2009).

Em anos recentes observou-se um incremento de estudos etnobotânicos preocupados em conhecer a cultura e o dia a 
dia de uma comunidade específica, ressaltando os conceitos locais de doença e saúde, além do modo como de como a comunidade utiliza os recursos naturais para a cura e prevenção de suas enfermidades (Patzlaff \& Peixoto, 2009). Alguns trabalhos podem ser citados, visando a preocupação dos autores em definir as prováveis espécies medicinais utilizadas em cada região, bem como o modo de manipulação destas, visando propriedades medicinais curativas e/ou preventivas, auxiliando na busca por novos compostos bioativos, juntamente com a

\section{Materiais e Métodos}

\section{Área de estudo}

O levantamento etnobotânico foi realizado no município de Hulha Negra - RS (Figura 1), que está localizado na região da Campanha, distando $374 \mathrm{Km}$ da capital do estado, Porto Alegre, com limites entre os municípios de Bagé e Candiota, com área geográfica de aproximadamente $822 \mathrm{~km}^{2}$ e uma população de 6.043 mil habitantes. O município de Hulha Negra é dividido na área urbana, onde se situa a sede do município, com 2.909 mil habitantes, e na área rural, formada pela Trigolândia e Assentamentos, análise do conhecimento popular brasileiro (Neto et al. 2014; Messias et al. 2015; Oliveira \& Lucena, 2015; Cruz et al. 2015; Leite et al. 2015; Araujo \& Lemos, 2015).

Considerando a necessidade de ampliar o conhecimento sobre a utilização de plantas medicinais em localidades com diversificação cultural e de elevada biodiversidade, o objetivo deste estudo foi verificar o perfil de utilização de plantas medicinais por indivíduos inseridos em um município com abrangência no Bioma Pampa e de colonização predominante alemã.

representando ao todo 3.134 mil habitantes (IBGE, 2010).

No século XVIII o município foi região muito disputada por índios, portugueses e espanhóis, sendo local de grande exploração de reservas de carvão. Com a chegada dos imigrantes alemães em 1925, a base econômica local passou a ser representada fortemente pela agricultura familiar, principalmente pela produção de trigo, e também na pecuária de gado de corte e leiteiro (Nunes, 2011). Atualmente, cerca de $20 \%$ da população é composta por pessoas de origem alemã (IBGE, 2010). 
FIGURA 1- Localização dos Bioma Pampa e Bioma de Mata Atlântica: (A) visão geral da América do Sul, (B) representação do Brasil e a classificação oficial dos Biomas brasileiros, (C) Localização do Município de Hulha Negra no estado do RS, inserido no Bioma Pampa.

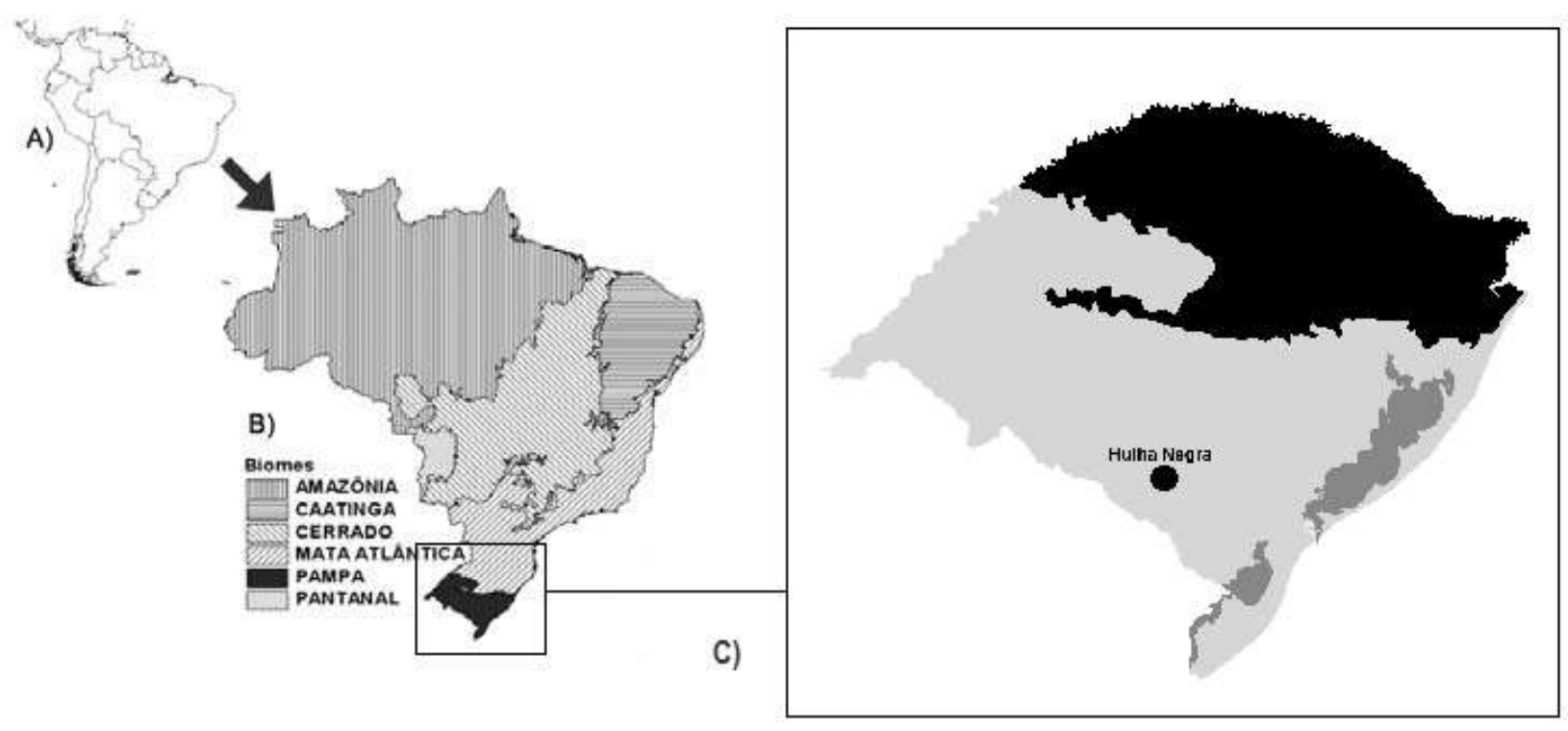

Fonte: Adaptação Overbeck, et al. (2009).

\section{População estudada e amostra}

Por questões de logística para a população-alvo deste estudo foram incluidos os indivíduos residentes no perímetro urbano do município (2.909 mil habitantes), localizados em seus domicílios. Para cálculo de amostra considerou-se prevalência estimada de consumo de plantas medicinais igual a 50\%, nível de confiança de $95 \%$ e erro aceitável de 5 pontos percentuais, sendo gerado a partir do software EpiInfo tamanho amostral no valor de $\mathrm{N}=344$ indivíduos. A escolha dos domicílios no perímetro urbano ocorreu de forma alternada para assegurar maior abrangência dos mesmos. Foi abordado somente um representante de cada domicílio com idade superior a dezoito anos.

\section{Coleta e análise de dados}

Através de delineamento observacional descritivo foram realizadas visitas domiciliares, compreendidas entre os dias úteis da semana, em horário comercial local (9h-12 e 14h-18h) durante os meses de agosto a novembro do ano de 2011. Foi utilizado um questionário estruturado abrangendo questões sócio-demográficas (sexo, idade e anos de estudo) da população e questões norteadoras sobre utilização de plantas medicinais (tipo de planta utilizada e motivo para uso).

As coletas botânicas foram realizadas à medida em que a população mencionava a aplicação da planta medicinal para a sua condição de saúde. Plantas cultivadas em 
domicílio ou provenientes dos arredores das residências dos moradores foram coletadas e/ou fotografadas. Ainda registrou-se informações relevantes para 0 reconhecimento das espécies a partir do relato dos depoentes. As identificações das plantas medicinais foram realizadas à partir da comparação do material coletado, fotografado e relatado pelos moradores, com a literatura especializada e comparação com espécimes depositados no Herbário Nicanor Risch da URCAMP.

\section{Resultados e Discussão}

Durante o período de coleta dos dados foi possível acessar 137 pessoas nos domicílios, pois muitos não se encontravam em suas residências pela dificuldade de seu acesso em horário comercial. Do total de entrevistados 93\% (128) relataram apresentar o hábito de utilizar plantas medicinais em seu cotidiano. Outros estudos etnobotânicos realizados em municípios do interior, também evidenciaram elevada prevalência de utilização de plantas medicinais por seus moradores com finalidades curativas/medicinais estando as mesmas representadas por 95,6\% (Dias et al., 2007); 90\% (Messias et al. 2015) e 73,51\% (Cruz et al., 2015), corroborando com os resultados encontrados no presente levantamento.

A elevada prevalência de utilização de plantas medicinais pode ser explicada pela
A categorização dos problemas de saúde a partir do motivo de uso das plantas medicinais à partir das falas do entrevistados realizada por sistemas orgânicos. Além disso, as espécies relatadas neste estudo foram agrupadas em plantas Nativas do Bioma Pampa, representadas por aquelas de crescimento espontâneo na região de abrangência do bioma em questão, e exóticas ao bioma, sendo estas oriundas de outras regiões ou até mesmo continentes.

facilidade de obtenção das mesmas no ambiente natural, as quais crescem espontaneamente nos arredores dos domicílios por estarem adaptadas a forma de cultivo às condições ambientais e climáticas da região. Ainda, a forte adesão de utilização de plantas medicinais no município de Hulha Negra pode estar justificada pela simplicidade de uso dos recursos naturais, seja sob a forma de infusos ou decoctos orientada pelo saber popular; pela falta de recursos financeiros para aquisição de medicamentos e pelo entendimento de que a utilização de plantas medicinais levam a menores efeitos colaterais em comparação aos medicamentos industrializados (Koch, 2000; Rodrigues \& Carvalho, 2001; Dias et al., 2007).

Através da análise do perfil dos usuários de plantas medicinais, é possível 
observar (Tabela 1) que dos 128

$(39,8 \%)$ com idade superior a 50 anos e 60 entrevistados, $89(69,5 \%)$ são mulheres, 51

$(46,8 \%)$ com nove ou mais anos de estudo.

TABELA 1- Descrição do perfil de usuários de plantas medicinais na localidade do município de Hulha Negra-RS. N= $128,2011$.

\begin{tabular}{lcc}
\hline Variável & N (128) & \% (100) \\
\hline Sexo & 89 & $\mathbf{6 9 , 5 \%}$ \\
\hline Mulheres & 39 & $\mathbf{3 0 , 5 \%}$ \\
Homens & & \\
Idade (anos) & 25 & $\mathbf{1 9 , 6 \%}$ \\
$\mathbf{2 0}-\mathbf{2 9}$ & 22 & $\mathbf{1 7 , 2 \%}$ \\
$\mathbf{3 0}-\mathbf{3 9}$ & 30 & $\mathbf{2 3 , 4 \%}$ \\
$\mathbf{4 0}-\mathbf{5 0}$ & 51 & $\mathbf{3 9 , 8 \%}$ \\
$\mathbf{5 0}$ ou mais & & \\
\hline Escolaridade (anos de estudo) & 18 & $\mathbf{1 4 , 1 \%}$ \\
\hline $\mathbf{0}-\mathbf{4}$ & 50 & $\mathbf{3 9 , 1 \%}$ \\
$\mathbf{5}-\mathbf{8}$ & & $\mathbf{4 6 , 8 \%}$ \\
\hline $\mathbf{9}-$ ou mais & $\mathbf{6 0}$ & \\
\hline
\end{tabular}

Fonte: primária.

A predominância do sexo feminino pode ter sido influenciada pelo período das entrevistas ter sido durante a semana em horário comercial, momento que geralmente na região os homens estão no trabalho e a maioria das mulheres de regiões interioranas permanecerem em casa cuidando dos afazeres domésticos. Entretanto, existem relatos que justificam a predominância da mulher no consumo de plantas medicinais, considerando a sua importância na transmissão do conhecimento entre as gerações e a responsabilidade pela execução do cuidado em saúde na família. (Schwabach, 2007;
Gomes, Nascimento \& Araúdo, 2007; Ceolin et al., 2011; Oliveira \& Meninni Neto, 2012).

Em relação a faixa etária, vários estudos relatam um aumento na frequência para uso de plantas medicinais a medida em que ocorre o aumento da idade, inferindo também aumento dos problemas de saúde com o passar dos anos, além disso outro fato preponderante nesta faixa etária, é o conhecimento adquirido de antepassados (Oliveira \& Meninni Neto, 2012; Messias et al., 2015; Oliveira \& Lucena, 2015). Considerando as informações relacionadas ao nível de escolaridade, podemos perceber que a população tem relativo grau de instrução, sendo este relato também evidenciado em 
estudo de Oliveira \& Lucena (2015), mostrando que o consumo de plantas medicinais independe da escolaridade.

Neste trabalho foram identificadas 67 diferentes espécies de plantas medicinais distribuídas em 28 famílias (Tabela 2). As famílias botânicas com mairo representatividade foram Asteraceae (21\%) e Lamiaceae $(16 \%)$, seguidas de Myrtaceae (7\%) e Apiaceae (6\%). Esse achado é muito similar a vários outros estudos realizados em municípios do interior dos estados do Rio
Grande do Sul e Minas Gerais em que a frequência de utilização para a família Asteraceae esteve entre $18 \%$ a $13 \%$ e Lamiaceae entre $17 \%$ a $14 \%$, demonstrando que nessas regiões as plantas medicinais destas famílias tem forte influência cultural (Marodin \& Baptista, 2001; Dias et al., 2007; Oliveira \& Meninni Neto, 2012, Alves \& Povh, 2013; Oliveira \& Lucena, 2015). Ainda, esse achado pode ser justificado pela elevada adaptabilidade das espécies nestas localidades (Oliveira \& Lucena, 2015; Messias et al. 2015).

TABELA 2. Descrição de Família, Nome Popular, Nome Científico, Motivo de Uso pelos Entrevistados, Procedência da Espécie e Número de Citações de cada espécie. N=128. Hulha Negra-RS, 2011.

\begin{tabular}{|c|c|c|c|c|c|c|}
\hline Família & & $\begin{array}{l}\text { Nome } \\
\text { Popular }\end{array}$ & Nome Científico & Usos Popular & Procedência & N.C \\
\hline 1 & Anacardiaceae & Anacauita & Schinus molle L. & Bronquite & Nativa & 1 \\
\hline 2 & Apiaceae & Aipo & Apium graveolens $\mathrm{L}$. & Febre & Exótica & 4 \\
\hline 3 & Apiaceae & $\begin{array}{l}\text { Anis } \quad \text { (erva- } \\
\text { doce })\end{array}$ & Pimpinella anisum L. & $\begin{array}{l}\text { Cólicas estomacais; } \\
\text { Calmante }\end{array}$ & Exótica & 4 \\
\hline 4 & Apiaceae & Funcho & $\begin{array}{l}\text { Foeniculum vulgare } \\
\text { Mill. }\end{array}$ & $\begin{array}{l}\text { Cólicas Estomacais; } \\
\text { Analgésico }\end{array}$ & Exótica & 10 \\
\hline 5 & Apiaceae & Salsa & $\begin{array}{l}\text { Petroselinum sativum } \\
\text { Hoffman }\end{array}$ & Diurético & Exótica & 4 \\
\hline 6 & Asteraceae & Alcachofra & Cynara scolymus L. & $\begin{array}{l}\text { Hipertensão arterial; } \\
\text { Emagrecimento }\end{array}$ & Exótica & 9 \\
\hline 7 & Asteraceae & Alcanfor & Artemisia alba Turra & Dores em geral & Exótica & 3 \\
\hline 8 & Asteraceae & $\begin{array}{l}\text { Arnica do } \\
\text { Campo }\end{array}$ & $\begin{array}{l}\text { Solidago microglossa } \\
\text { DC. }\end{array}$ & $\begin{array}{l}\text { Anti-inflamatório; } \\
\text { Cicatrizante }\end{array}$ & Nativa & 11 \\
\hline 9 & Asteraceae & Aspirina & Achillea millefolium L. & Cefaleias & Exótica & 2 \\
\hline 10 & Asteraceae & Camomila & $\begin{array}{l}\text { Matricaria chamomilla } \\
\text { L. }\end{array}$ & Insônia; Calmante & Exótica & 11 \\
\hline 11 & Asteacea & Carqueja & $\begin{array}{l}\text { Baccharis trimera } \\
\text { (Less.) A.P. de Candolle }\end{array}$ & $\begin{array}{l}\text { Emagrecimento; Dores em } \\
\text { geral }\end{array}$ & Nativa & 15 \\
\hline
\end{tabular}




\begin{tabular}{|c|c|c|c|c|c|c|}
\hline 12 & Asteraceae & $\begin{array}{l}\text { Carqueja } \\
\text { branca }\end{array}$ & $\begin{array}{l}\text { Baccharis articulata } \\
\text { (Lam.) Pers. }\end{array}$ & Emagrecimento; Digestivo & Nativa & 6 \\
\hline 13 & Asteraceae & Dente-de-leão & Taraxacum officinale $\mathrm{L}$. & Anti-inflamatório & Exótica & 1 \\
\hline 14 & Asteraceae & Guaco & glomerata & Bronquite; Analgésico & Nativa & \\
\hline 15 & Asteaceae & Infalivina & $\begin{array}{l}\text { Artemisia verlotorum } \\
\text { Lamotte }\end{array}$ & Problemas hepáticos & Exótica & 1 \\
\hline 16 & Asteraceae & Losna & $\begin{array}{l}\text { Artemisia absinthium } \\
\text { (Mill.) DC. }\end{array}$ & Digestivo & Exótica & 5 \\
\hline 17 & Asteraceae & Macela & $\begin{array}{l}\text { Achyrocline satureioides } \\
\text { (Lam.) DC. }\end{array}$ & $\begin{array}{l}\text { Hipertensão arterial; } \\
\text { Diurético }\end{array}$ & Nativa & 55 \\
\hline 18 & Asteraceae & Mil Folhas; & Achillea millefolium $\mathrm{L}$. & Cólicas menstruais & Exótica & 3 \\
\hline 19 & Asteraceae & Palma & Tanacetum vulgare $\mathrm{L}$. & $\begin{array}{l}\text { Cólicas estomacais; } \\
\text { Problemas hepáticos; Anti- } \\
\text { inflamatório }\end{array}$ & Exótica & 8 \\
\hline 20 & Brassicaceae & Agrião & $\begin{array}{l}\text { Nasturtium officinale } \\
\text { R.Br. }\end{array}$ & Tosse & Exótica & 9 \\
\hline 21 & Cactaceae & Tuna & $\begin{array}{l}\text { Cereus hildmannianus } \\
\text { K. Schum. }\end{array}$ & Coqueluche & Nativa & 1 \\
\hline 22 & Celastraceae & $\begin{array}{l}\text { Espinheira } \\
\text { Santa }\end{array}$ & $\begin{array}{l}\text { Maytenus ilicifolia Mart. } \\
\text { Ex Reissek }\end{array}$ & Gastrite & Nativa & 8 \\
\hline 23 & Fabaceae & Pata de vaca & Bauhinia forficata $\mathrm{L}$. & $\begin{array}{l}\text { Diabetes; Problemas } \\
\text { renais; Diurético }\end{array}$ & Nativa & 10 \\
\hline 24 & Fabaceae & Sene & Cassia angustifolia Vahl & Laxante; Emagrecimento & Exótica & 4 \\
\hline 25 & Juglandaceae & Nogueira & Juglans regia $\mathrm{L}$. & $\begin{array}{l}\text { Anticolesterolêmico; } \\
\text { Diabetes }\end{array}$ & Exótica & 2 \\
\hline 26 & Lamiaceae & Alfazema & $\begin{array}{l}\text { Lavandula officinalis } \\
\text { Chaix }\end{array}$ & Anestésico & Exótica & 3 \\
\hline 27 & Lamiaceae & Alecrim & $\begin{array}{l}\text { Rosmarinus officinalis } \\
\text { L. }\end{array}$ & $\begin{array}{l}\text { Hipertensão arterial; } \\
\text { Diurético }\end{array}$ & Exótica & 5 \\
\hline 28 & Lamiaceae & Alevante & Mentha citrata Ehrh. & $\begin{array}{l}\text { Estimulante do Sistema } \\
\text { Nervoso Central }\end{array}$ & Exótica & 1 \\
\hline 29 & Lamiaceae & Falso Boldo & $\begin{array}{l}\text { Plectranthus barbatus } \\
\text { Andr. }\end{array}$ & $\begin{array}{l}\text { Estimulante do Sistema } \\
\text { Nervoso Central; Digestivo }\end{array}$ & Exótica & 22 \\
\hline 30 & Lamiaceae & Colé & $\begin{array}{l}\text { Coleus forskohlii } \\
\text { (Willd.) Briq. }\end{array}$ & Anticolesterolêmico & Exótica & 2 \\
\hline 31 & Lamiaceae & Hortelã & Mentha $\mathrm{X}$ piperita $\mathrm{L}$. . & $\begin{array}{l}\text { Analgésico; } \\
\text { Antiespasmódico; } \\
\text { Digestivo }\end{array}$ & Exótica & 13 \\
\hline & Lamiaceae & Maragatinha & $\begin{array}{l}\text { Salvia coccínea Juss. ex } \\
\text { Murray }\end{array}$ & Digestivo; Cefaleia & Exótica & 2 \\
\hline
\end{tabular}




\begin{tabular}{|c|c|c|c|c|c|c|}
\hline & & & & & Exótica & \\
\hline 33 & Lamiaceae & Melissa & Melissa officinalis L. & Calmante & & 8 \\
\hline 34 & Lamiaceae & $\begin{array}{l}\text { Orelha-de- } \\
\text { coelho }\end{array}$ & Stachys lanata Jacq. & Próstata; Problemas renais & Exótica & 2 \\
\hline 35 & Lamiaceae & Poejo & $\begin{array}{l}\text { Cunila microcephala } \\
\text { Benth. }\end{array}$ & $\begin{array}{l}\text { Analgésico; Anti- } \\
\text { inflamatório }\end{array}$ & Nativa & 10 \\
\hline 36 & Lamiaceae & Sálvia & Salvia officinalis $\mathrm{L}$. & Bronquite; Analgésico & Exótica & 6 \\
\hline 37 & Lauraceae & Abacateiro & Persea americana Mill. & $\begin{array}{l}\text { Diurético; Hipertensão } \\
\text { arterial }\end{array}$ & Exótica & 3 \\
\hline 38 & Lauraceae & Canela & $\begin{array}{l}\text { Cinnamomum } \\
\text { zeylanicum Blume }\end{array}$ & $\begin{array}{l}\text { Diabetes; Hipertensão } \\
\text { arterial }\end{array}$ & Exótica & 2 \\
\hline 39 & Lythraceae & Romã & Punica granatum L. & Antidiarreico & Exótica & 6 \\
\hline 40 & Malvaceae & Guanxuma & Sida rhombifolia $\mathrm{L}$. & Digestivo & Nativa & 2 \\
\hline 41 & Malvaceae & Malva & Malva sylvestris L. & Anti-inflamatório & Exótica & 14 \\
\hline 42 & Moraceae & Amora & Morus nigra $\mathrm{L}$. & Gastrite, Diabetes & Exótica & 2 \\
\hline 43 & Moraceae & Figueira & Ficus carica $\mathrm{L}$. & Antidiarreico ; Analgésico & Exótica & 2 \\
\hline 44 & Myrtaceae & Araçá & $\begin{array}{l}\text { Psidium cattleyanum var } \\
\text { coriaceum (Mart. ex O. } \\
\text { Berg) Kiaersk }\end{array}$ & Diabetes & Nativa & 1 \\
\hline 45 & Myrtaceae & Eucalipto & $\begin{array}{l}\text { Eucalyptus globulus } \\
\text { Labill. }\end{array}$ & $\begin{array}{l}\text { Bronquite; } \\
\text { Descongestionante nasal }\end{array}$ & Exótica & 4 \\
\hline 46 & Myrtaceae & Jambolão & $\begin{array}{l}\text { Syzygium cumini (L.) } \\
\text { Skeels }\end{array}$ & Diabetes & Exótica & 4 \\
\hline 47 & Myrtaceae & Murta & $\begin{array}{l}\text { Blepharocalyx } \\
\text { salicifolius (Kunt) O. } \\
\text { Berg. }\end{array}$ & Digestivo & Nativa & 14 \\
\hline 48 & Myrtaceae & Pitangueira & Eugenia uniflora $\mathrm{L}$. & $\begin{array}{l}\text { Calmante; Digestivo; } \\
\text { Antidiarreico }\end{array}$ & Nativa & 6 \\
\hline 49 & Oleaceae & Oliveira & Olea europaea L. & Emagrecimento & Exótica & 2 \\
\hline 50 & Passifloraceae & Maracujá & Passiflora caerulea $\mathrm{L}$. & $\begin{array}{l}\text { Calmante; Emagrecimento; } \\
\text { Diurético }\end{array}$ & Nativa & 3 \\
\hline 51 & Phyllanthaceae & Quebra-pedra & Phyllanthus niruri L. & $\begin{array}{l}\text { Diurético; Problemas } \\
\text { renais }\end{array}$ & Nativa & 8 \\
\hline 52 & Plantaginaceae & Tanchagem & Plantago australis Lam. & $\begin{array}{l}\text { Anti-inflamatório; } \\
\text { Diurético }\end{array}$ & Nativa & 8 \\
\hline 53 & Poaceae & Capim cidreira & $\begin{array}{l}\text { Cymbopogon citratus } \\
\text { (DC.)Stapf }\end{array}$ & $\begin{array}{l}\text { Calmante; Hipertensão } \\
\text { arterial, Analgésico }\end{array}$ & Exótica & 15 \\
\hline 54 & Polygonaceae & Erva-de-bicho & $\begin{array}{l}\text { Polygonum } \\
\text { hydropiperoides Michx. }\end{array}$ & Anti-inflamatório & Exótica & 1 \\
\hline 55 & Rhamnaceae & Uva do Japão & Hovenia dulcis Thunb. & Anticolesterolêmico & Exótica & 1 \\
\hline
\end{tabular}




\begin{tabular}{|c|c|c|c|c|c|c|}
\hline \multirow[b]{2}{*}{56} & \multirow[b]{2}{*}{ Rhamnaceae } & \multirow[b]{2}{*}{ Coronilha } & \multirow[b]{2}{*}{ Scutia buxifolia Reiss } & \multirow[b]{2}{*}{ Hipertensão arterial } & \multicolumn{2}{|l|}{ Nativa } \\
\hline & & & & & & 1 \\
\hline & & & & & Exótica & \\
\hline 57 & Rosaceae & Morango & Fragaria $X$ ananassa $L$. & Digestivo & & 1 \\
\hline & & & & & Nativa & \\
\hline 58 & Rutaceae & Bergamoteira & Citrus reticulata Blanco & Analgésico & & 2 \\
\hline 59 & Rutaeae & Laranjeira & $\begin{array}{l}\text { Citrussinensis } \quad(\quad \text { L. }) \\
\text { Osbeck }\end{array}$ & Dores em geral; Analgésico & Nativa & 9 \\
\hline 60 & Rutaceae & Lima & Citrus $x$ aurantium L. & Analgésico & Nativa & 2 \\
\hline & & & & & Nativa & \\
\hline 61 & Salicaceae & Erva-de-bugre & Casearia sylvestris $\mathrm{Sw}$. & Depurativo & & 1 \\
\hline 62 & Santalaceae & $\begin{array}{l}\text { Cancorosa de } \\
\text { três espinhos }\end{array}$ & $\begin{array}{l}\text { Jodinarhombifolia } \\
\text { Hook. Et. Arn. }\end{array}$ & Digestivo & Nativa & 2 \\
\hline 63 & Theaceae & Chá verde & $\begin{array}{l}\text { Camellia sinensis (L.) } \\
\text { Kuntze }\end{array}$ & Emagrecimento; Digestivo & Exótica & 5 \\
\hline 64 & Verbenaceae & Cidrão & $\begin{array}{l}\text { Aloysia citriodora } \\
\text { Ortega ex Pers. }\end{array}$ & Calmante & Nativa & 8 \\
\hline 65 & Verbenaceae & $\begin{array}{l}\text { Erva } \\
\text { carniceira }\end{array}$ & $\begin{array}{l}\text { Aloysia triphylla } \\
\text { (L'Hér.) Britton }\end{array}$ & Gastrite & Nativa & 1 \\
\hline 66 & Verbnaceae & Gervão & $\begin{array}{l}\text { Stachytarphera } \\
\text { cayennensis (Rich.) Vahl }\end{array}$ & Emagrecimento; Digestivo & Nativa & 3 \\
\hline 67 & $\begin{array}{l}\text { Xanthorrhoeae } \\
\text { ae }\end{array}$ & Babosa & Aloe barbadensis Mill. & Cicatrizante & Exótica & 4 \\
\hline
\end{tabular}

Embora ainda sejam escassos os estudos com as plantas medicinais no Bioma Pampa, alguns relatos vem mostrando espécies que vêm sendo exploradas para uso medicinal nas práticas de cuidado em saúde em comunidades, podendo ser destacada a macela (Achyrocline satureioides), bananinha-domato (Bromelia antiacantha), carqueja (Baccharis trimera), espinheira-santa (Maytenus ilicifolia), erva-de-bugre (Casearia sylvestris), coronilha (Scutia buxifolia) e insulina-do-mato (Sphagneticola trilobata) (Rodrigues et al., 2007; Ceolin, 2011).

Neste estudo as espécies mais citadas pelos entrevistados foram a Macela
[Achyrocline satureioides (Lam.) D.C.] com 55 citações, seguida pelo Falso Boldo [Plectranthus barbatus Andr.] (22); Capim Cidreira [Cymbopogon citratus (DC.) Stapf] (15); Carqueja [Baccharis trimera (Less.) D.C.] (15); Murta [Blepharocalyx salicifolius (Kunt) O. Berg.] (14), Malva [Malva sylvestris L.] (14), Hortelã [Mentha piperita L] (13), além da Arnica do Campo [Solidago microglossa DC.] (11) e Camomila [Matricaria chamomilla L.] (11). Dias et al. (2007); Oliveira \& Lucena (2015) e Messias et al. (2015) também encontraram similaridade na frequencia de uso para as mesmas plantas. 
Considerando a frequência de citação da Macela (Achyrocline satureioides (Lam.) D.C.) e do Falso Boldo (Plectranthus barbatus Andr.), pode-se justificar os principais motivo de uso pela população estudada, sendo os mesmos, associados ao sistema digestivo e as condições de dor e inflamação (Figura 2). Morbidades crônicas como problemas cardiovasculares e condição de diabetes, e situações envolvendo o comprometimento do trato respiratório e urinário também motivaram o uso de plantas medicinais, estando estes achados em concordância com os estudos de Oliveira \& Menini Neto (2012) e Dias et al. (2007). Corroborando, Silva \& Hahn (2011) descrevem que as principais enfermidades com utilização de plantas medicinais para fins terapêuticos no Rio Grande do Sul são as situações que acometem o sistema digestivo, a hipertensão arterial, diabetes mellitus e dislipidemias, encontrando uma prevalência de utilização de plantas medicinais igual a 62,4\%. Ainda, para estas condições de saúde, também evidenciaram a Macela (Achyrocline satureioides) como a espécie mais utilizada. Segundo López-Jaramillo et al. (2005) os países latino-americanos vem cada vez mais aumentando suas estimativas para as desordens envolvendo os sistema cardiovascular e morbidades correlacionadas como diabetes mellitus e a síndrome metabólica, o que impulsiona o uso de plantas medicinais, pois estes países, assim como no Brasil, sofrem forte influência cultural para uso de espécies vegetais como método de tratamento para os problemas de saúde.

FIGURA 2- Frequência das plantas medicinais citadas conforme classificação por sistemas orgânicos.

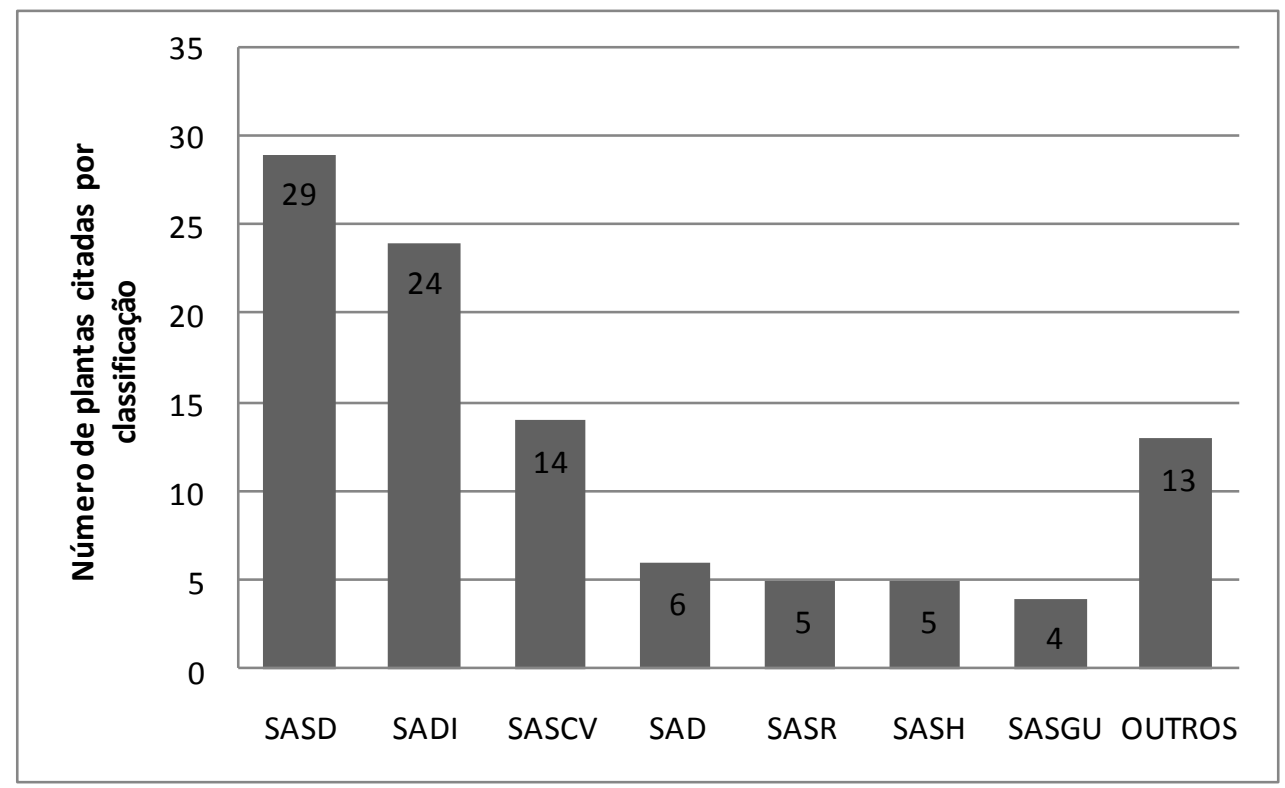

Legenda: $\mathrm{SASD}=$ sintomas associados ao sistema digestivo; $\mathrm{SADI}=$ sintomas associados a dor e inflamação; $\mathrm{SASCV}=$ sintomas associados ao sistema cardiovascular; $\mathrm{SAD}=$ sintomas associados ao diabetes; $\mathrm{SASR}=$ sintomas associados ao sistema respiratório; $\mathrm{SASH}=$ sintomas associados ao sistema hepático; SASGU= sintomas associados ao sistema gênitourinário; OUTROS= sintomas associados a/ou cicatrização, calmante, coqueluche, sistema nervoso central, processos depurativos. Fonte: Primária. 
A região sul do Brasil apresenta uma riqueza de vegetais cultivados compreendendo frutíferas, ornamentais, forrageiras e medicinais em decorrência da colonização do território por diferentes etnias (indígenas, portugueses, espanhóis, africanos, alemães, italianos e franceses) , o que possibilitou a existência de uma diversidade de variedades locais, adaptadas ás condições locais. Conforme Lorenzi e Abreu Matos (2002) os conhecimentos sobre a flora local acabaram-se fundindos aqueles trazidos da Europa, muitas vezes de uso popular bastante difundido. Além disso, muitas plantas conhecidas no Velho mundo por suas propriedades medicinais induziram os europeus a testarem usos similares as espécies nativas proximamente relacionadas. Muitas vezes, o mesmo princípio podia ser encontrado nas espécies nativas, ocasionalmente em maior quantidade e qualidade.

Das 67 espécies identificadas, 26 $(38,8 \%)$ correspondem a plantas nativas da América do Sul, as quais encontram-se naturalmente no Bioma Pampa. Entretanto, 41 $(61,2 \%)$ são classificadas como exóticas (Tabela 1). Esses dados são fundamentados

\section{REFERÊNCIAS}

ARAUJO, J.L.; LEMOS, J.R. Estudo etnobotânico sobre plantas medicinais na comunidade de Curral Velho, Luís Correia, Piauí, Brasil. Biotemas, v.28, n.2, p.125-136, 2015. por Koch (2000) onde afirma que grande parte das plantas cultivadas são de origem européia devido à imigração, em especial alemã e italiana que ocorreu em diversas regiões brasileiras, estando estas espécies adaptadas às condições ambientais e climáticas do estado do Rio Grande do Sul, sendo algumas cultivadas e outras fazendo parte da vegetação espontânea, formando o ambiente natural. Também Messias et al. (2015), relatam em seu estudo realizado no estado de Minas Gerais, que das 342 espécies citadas, $40 \%$ eram exóticas, sobressaindo também, as de origem européia.

Com base nesses achados, mesmo não tendo sido acompanhada o total da população do cálculo de amostra, podemos inferir que a população correspondente ao município de Hulha Negra-RS possui um importante repertório sobre plantas medicinais, o que colabora para estudos sobre a diversidade etnobotânica da região, visto que faz-se uso de um grande acervo botânico com intuito de prevenir e curar problemas de saúde na comunidade, abrindo também, um importante espaço para um processo de cuidado $\mathrm{e}$ educação em saúde para plantas medicinais do Bioma Pampa brasileiro. 
CEOLIN, T.; HECK, M.R.; BARBIERI, R.L.; SCHWARTZ, E.; MUNIZ, R.M.; PILLON, C. N. Plantas Medicinais: transmissão do conhecimento nas famílias de agricultores de base ecológica no Sul do RS. Revista da Escola de Enfermagem da USP, v.45, n.1, p.47-54, 2011.

CRUZ, M.J.B.; DOURADO, L.F.N.; BODEVAN, E.C.; ARAÚJO, L.U.; GRAEL, C.F.F.; SANTOS, D.F. Uso de Plantas Medicinais por Famílias do Vale do Jequitinhonha, Minas Gerais, Brasil. InfarmaCiências Farmacêuticas, v.27, n.1, p.38-48, 2015.

DIAS, I.M.S.B.; SARMENTO, M.B.; SOUZA, R.G.; PEREIRA, M.P.

Levantamento Etnobotânico em Seis Municípios da Região da Campanha, RS. Revista Científica Rural, v.12, n.1, p.114-130, 2007.

GIULIETTI, A.M.; HARLEY, R.M.; QUEIROZ, L.P.; WANDERLEY, M.G.L; VAN DER BERG, C. Biodiversity and conservation of plants in Brazil. Conservation Biology. v.19, n. 3, p.632-639, 2005.

GOMES, R.; NASCIMENTO, E.F.; ARAÚJO, F.C. Por que os homens buscam menos serviços de saúde do que as mulheres? As explicações de homens de baixa escolaridade e homens com ensino superior. Caderno de Saúde Pública, v.23, n.3, p.565574, 2007.

IBGE, Instituto Brasileiro de Geografia e Estatística, Comunicação pessoal, Hulha Negra: 2010.

KRUEL, V.S.F.; SILVA, I.M; PINHEIRO, C.U.B. O Ensino Acadêmico da Etnobotânica no Brasil. Rodriguésia, v.56, n.87, p.97-106, 2005.

KOCH, V. Estudo Etnobotânico das Plantas Medicinais na Cultura Ítalo-brasileira no Rio Grande do Sul. 2000 - 138p. Dissertação (Mestrado em Fitotecnia) - Departamento de Fitotecnia, Universidade Federal do Rio Grande do Sul, Porto Alegre.
LEITE, I.A.; MORAIS, A.M.; DO Ó, K.D.S.; CARNEIRO, R.G.; LEITE, C.A. A Etnobotânica De Plantas Medicinais No Município De São José De Espinharas, Paraiba, Brasil. Biodiversidade, v.14, n.1, p.22, 2015.

LÓPEZ-JARAMILLO, P.; SÁNCHEZ, R.A.; DIAZ, M.; COBOS, L.; BRYCE, A.; PARRA-CARRILLO, J.Z.; LIZCANO, F.; LANAS, F.; SINAY, I.; SIERRA, I.D.; PEÑAHERRERA, E.; BENDERSKY, M.; SCHMID, H.; BOTERO, R.; URINA, M.; LARA, J.; FOSS, M.C.; MÁRQUEZ, G.; HARRAP, S.; RAMÍREZ, A.J.;

ZANCHETTI, A. Consenso latino-americano de hipertensão em pacientes com diabetes tipo 2 e síndrome metabólica. Arquivos Brasileiros de Endocrinologia Metabolica. v.58, n.3, p.225-225, 2005.

\section{LORENZI, H.; MATOS, F.J.A.; Plantas}

Medicinais no Brasil: nativas e exóticas. 2.ed. Nova Odessa (SP): Instituto Plantarum, 2008, $544 \mathrm{p}$.

MARODIN, S.M. Plantas Utilizadas como Medicinais no Município de Dom Pedro de Alcântara, Rio Grande do Sul. 2001 - 112p. Dissertação (Mestrado em Botânica) Departamento de Botânica, Universidade Federal do Rio Grande do Sul, Porto Alegre.

MESSIAS, M.C.T.B.; MENEGATTO, M.F.; PRADO, A.C.C.; SANTOS B.R.; GUIMARÃES, M.F.M. Uso popular de plantas medicinais e perfil socioeconômico dos usuários: um estudo em área urbana em Ouro Preto, MG, Brasil. Revista Brasileira de Plantas Medicinais, v.17, n.1, p.76-104, 2015.

NETO, F.R.G.; ALMEIDA, G.S.S.A.; JESUS, N.G.; FONSECA, M.R. Estudo Etnobotânico de plantas medicinais utilizadas pela Comunidade do Sisal no município de Catu, Bahia, Brasil. Revista Brasileira de Plantas Medicinais, v.16, n.4, p.856-865, 2014.

NUNES, J.J.D.A. Atividade leiteira e agricultura familiar de Hulha Negra-RS: limitações e potencialidades da Cooperativa 
Agrícola Mista Aceguá para o

desenvolvimento local. - $2011-58$ p.

Dissertação (Mestrado em Ciências

Econômicas ) - Universidade Federal do Rio

Grande do Sul, Porto Alegre.

OLIVEIRA, D.M.S.; LUCENA, E.M.P. O uso de plantas medicinais por moradores de Quixadá-Ceará. Revista Brasileira de Plantas Medicinais, v.17, n.3, p.407-412, 2015.

OLIVEIRA, E.R.; MENINI NETO, L. Levantamento etnobotânico de plantas medicinais utilizadas pelos moradores do povoado de Manejo, Lima Duarte - MG. Revista Brasileira de Plantas Medicinais, v.14, n.2, p.311-320, 2012.

OVERBECK, G.E.; MÜLLER, S.C.; FIDELIS, A.; PFADENHAUER, J.; PILLAR, V.P.; BLANCO, C.C.; BOLDRINI, I.L.; BOTH, R.; FORNECK, E.D. Os Campos Sulinos: um bioma negligenciado. In: Campos sulinos conservação e uso sustentável da biodiversidade. Ministério do Meio Ambiente, p.63-77, 2009.

PATZLAFF, R.G.; PEIXOTO, A.L. A pesquisa em etnobotânica e o retorno do conhecimento sistematizado à comunidade: um assunto complexo. Revista História, Ciências e Saúde, v.16 n.1, p.237-246, 2009.

RODRIGUES, V.E.G.; CARVALHO, D.A. Levantamento Etnobotânico de Plantas Medicinais do Domínio do Cerrado na Região do Alto Rio Grande - Minas Gerais. Revista Ciência e Agrotecnologia, v.25 n.1, p.102123, 2001.
RODRIGUES, W. F. ; GOMES, G. C. ; MEDEIROS, A. R. M. ; BARBIERI, R. L. Espécies arbóreas da serra dos Tapes: um resgate etnobotânico (Documentos 190). Pelotas: Embrapa Clima Temperado, p. 68, 2007. Disponível em:

<http://ainfo.cnptia.embrapa.br/digital/bitstrea m/item/40069/1/documento-190.pdf > . Acesso em: 20 out. 2015.

SILVA, B.Q.; HAHN, S.R. Uso De Plantas Medicinais Por Indivíduos Com Hipertensão Arterial Sistêmica, Diabetes Mellitus Ou Dislipidemias. R. Bras. Farm. Hosp. Serv. Saúde São Paulo, v.2, n.3, 36-40, 2011.

SIMÕES, C.M.O.; MENTZ, L.A.; SCHENKEL E.P.; IRGANG, B.E.; STEHMANN J.R.; Plantas da Medicina Popular no Rio Grande do Sul. 5.ed. Porto Alegre: Editora Universidade/UFRGS, 1998, $173 p$.

SCHWAMBACH, K.H. Utilização de Plantas Medicinais e Medicamentos no Autocuidado no município de Teutônia, RS. 2007, 97p.

Dissertação (Mestrado em Ciências Farmacêuticas) - Departamento de Ciências Farmacêuticas, Universidade Federal do Rio Grande do Sul, 2007.

TOMAZZONI, M.I.; NEGRELLE, R.R.B.; CENTA, M.L. Fitoterapia Popular, a Busca Instrumental Enquanto Prática Terapêutica. Texto e contexto de Enfermagem, v.15, n.1, p.115-121, 2006.

VEIGA JR, V.F.; PINTO, A.C.; MACIEL, M.A.M. Plantas Medicinais: Cura Segura? Química. Nova, v.28, n.3, p.519-528, 2005. 